Periodica Mathematica Hungarica Vol. 3 (3-4), (1973), pp. 235-241.

\title{
ON HEREDITARY RADICALS
}

\author{
by
}

F. SZÁSZ and R. WIEGANDT (Budapest)

0. In this paper we shall show that hereditary radical classes occur considerably frequently in the sense that to any cardinality $m$ there exist radical classes $\mathbf{P}, \mathbf{Q}$ such that exactly $2^{\mathfrak{m}}$ radieal classes $\mathbf{R}$ satisfy $\mathbf{P} \subseteq \mathbf{R} \subseteq \mathbf{Q}$, and all the radicals $\mathbf{R}$ are hereditary (Theorem 1 ). On the other hand example will be given for hereditary radical classes $\mathbf{P} \subseteq \mathbf{Q}$ such that there is no hereditary radical between $\mathbf{P}$ and $\mathbf{Q}$. To any radical class $\mathbf{R}$ there exists a unique maximal hereditary radical class $\mathbf{H}_{\mathbf{R}}$ with $\mathbf{H}_{\mathbf{R}} \subseteq \mathbf{R}$, and a unique minimal hereditary radical elass $\overline{\mathbf{R}}$ with $\mathbf{R} \subseteq \overline{\mathbf{R}}$. It will be investigated when an upper radical elass will be hereditary (Theorems 4,5 and 6 ).

1. All rings considered are associative. As it is well-known, a class $\mathbf{R}$ of rings is ealled a radical class in the sense of Kunoš-AmrTsur, if

( $R$ 1) $\mathbf{R}$ is homomorphically closed,

(R 2) every ring $A$ has an $\mathbf{R}$-ideal $\mathbf{R}(A)$ which contains all $\mathbf{R}$-ideals of $A, \mathbf{R}(A)$ is called the $\mathbf{R}$-radical of $A$,

$(\mathrm{R} 3)$ the factorring $A / \mathbf{R}(A)$ does not contain non-zero $\mathbf{R}$-ideals.

Sometimes radical classes will be briefly called radicals. $\mathbf{A}$ elass $\mathbf{K}$ of rings is said to be hereditary, if every ideal of a $\mathbf{K}$-ring is again a $\mathbf{K}$-ring. A semisimple class $\mathbf{S}$ means a class of rings having properties

(S 1) $\mathbf{S}$ is hereditary,

(S 2) if every non-zero ideal of a ring $A$ has a non-zero homomorphic image in $\mathbf{S}$, then $A$ is an $\mathbf{S}$-ring.

Now we recall some well-known facts and constructions of the radical theory. For details we refer to Divinsky [5].

To any hereditary class $\mathbf{M}$ the class $\overline{\mathbf{M}}$ defined as the class of all rings $A$ such that every non-zero ideal of $A$ can be mapped homomorphically onto some non-zero ring of $\mathbf{M}$, forms a semisimple class. If $\mathbf{R}$ is a radical class then the class of all rings having zero R-radical, forms a semisimple class which will be denoted by $\mathbf{S R}$. If $\mathbf{M}$ is a hereditary class, then the class of all rings which cannot be mapped homomorphically onto a non-zero ring of $\mathbf{M}$, is a radical class, the so called upper radical class of $\mathbf{M}$ and we shall denote it by $\mathbf{U M}$. 
To any homomorphically closed class $\mathbf{N}$ one can construct the lower radical $\mathbf{L N}$ as follows: Let $\mathbf{N}_{1}=\mathbf{N}$, and define $\mathbf{N}_{k}=$ \{every ring: every nonzero homomorphic image of which has a non-zero ideal in $\mathbf{N}_{l}$ for some $\left.l<k\right\}$ for each $k=2,3, \ldots$ Now let $\mathbf{L N}$ be $\mathbf{N}_{\omega}$ (cf. SULIŇSKI-ANDERSON-DrviNSKY [8]). If $\mathbf{N}$ is hereditary, then also the lower radical $\mathbf{L N}$ will be hereditary (ef. Armendariz-Leavite [4] and Watters [9]). A subring $B$ of a $\operatorname{ring} A$ is called an accessible subring if there is a chain $B=A_{n} \subset \ldots \subset A_{1}=A$ of subrings, such that each $A_{i+1}$ is an ideal in $A_{i}$ for $i=1, \ldots, n \ldots 1$.

If $\mathbf{P}$ and $\mathbf{Q}$ are radical classes then $[\mathbf{P}, \mathbf{Q}]$ will denote the class of all radicals $\mathbf{R}$ with $\mathbf{P} \subseteq \mathbf{R} \subseteq \mathbf{Q}$.

2. In this section we shall show that between two radical classes there may be relatively very many radical classes. For this sake let $\mathbf{F}$ be a class (but not a set ${ }^{1}$ ) of fields, and let UF denote the upper radical determined by $\mathbf{F}$, further consider a subset $\mathbf{F}(\mathrm{m}) \subseteq \mathbf{F}$ of cardinality $\mathrm{m}$.

THEOREM 1. Let $\mathbf{P}(\subseteq \mathbf{U F})$ be a radical class. If $\mathbf{Q}$ is the lower radical $\mathbf{L}(\mathbf{P} \cup \mathbf{F}(\mathfrak{m}))$ belonging to $\mathbf{P} \cup \mathbf{F}(\mathfrak{m})$, then $[\mathbf{P}, \mathbf{Q}]$ has exactly $2^{\mathrm{m}}$ radical classes, moreover, all radicals of $[\mathbf{P}, \mathbf{Q}]$ are hereditary if and only if $\mathbf{P}$ is hereditary.

Proof. Let $\mathbf{R}$ be a radical of $[\mathbf{P}, \mathbf{Q}]$. Consider the subset $\mathbf{F}(\mathfrak{n})$ consisting of all fields of $\mathbf{F}(\mathfrak{n})$ which are ideals in some rings of $\mathbf{R}$ (nd denotes the cardinality of $\mathbf{F}(\mathfrak{m})$ ). Let us remark that if $B \in \mathbf{F}(\mathfrak{n})$ and $B$ is an ideal of a ring $A$, then $A$ splits always in a direct sum $A=B \oplus C$, because $B$ is a ring with unity element. Hence $A \in \mathbf{R}$ implies also $B \in \mathbf{R}$. Thus $P \cup \mathbf{F}(\mathfrak{n}) \subseteq \mathbf{R}$ holds which implies $\mathbf{L}(\mathbf{P} \cup \mathbf{F}(\boldsymbol{\eta})) \subseteq \mathbf{R}$.

If $A$ is an $\mathbf{R}$-ring not belonging to $\mathbf{P}$, then the $\operatorname{ring} A_{1}=A / \mathbf{P}(A)$ is not zero and it has zero $\mathbf{P}$-radical, but $\mathbf{R}\left(A_{1}\right)=A_{1}$ holds. Since $\mathbf{Q}$ is a lower radical, so it is the union $\mathbf{Q}=\mathbf{N}_{\omega}$ where $\mathbf{N}_{1}=\mathbf{P} \cup \mathbf{F}(n)$ and $\mathbf{N}_{k}$ is defined as in Section 1. Hence $\mathbf{Q}\left(A_{1}\right)=A_{1}$ and so by Lemma 1 of [8] there exists an accessible subring $A_{n} \neq 0$ of $A_{1}$ such that $A_{n}$ belongs to $\mathbf{N}_{1}=\mathbf{P} \cup \mathbf{F}(\mathfrak{n})$. If $A_{n} \in \mathbf{P}$, then by Theorem 1 of Anderson-Divinsky-Sulińskr [1] the P-radical, $\mathbf{P}\left(A_{n-1}\right)$ is an ideal of $A_{n-2}$ and $0 \neq A_{n} \subseteq \mathbf{P}\left(A_{n-1}\right)$ holds. Hence also $A_{n-3}, \ldots, A_{1}$ have non-zero P-radicals which contradicts the choice of $A_{1}$. Thus $A_{1}$ has an accessible subring $A_{n} \in \mathbf{F}(\mathrm{m})$. Hence $A_{n}$ is a field, and according to ANDRUNAKIEvič lomma for the ideal $A_{n}^{*}$ generated by $A_{n}$ in $A_{1}$, we have $A_{n}=A_{n}^{3} \subseteq A_{n}^{* 3} \subseteq A_{n}$. Consequently $A_{n}$ is an ideal of $A_{1}$ which impliés $A_{n} \in \mathbf{F}(\mathfrak{i})$. Hence, clearly $\mathbf{R} \subseteq \mathbf{L}(\mathbf{P} \cup \mathbf{F}(\mathfrak{n}))$ follows.

Since $\mathbf{F}(\mathfrak{m})$ has exactly $2^{\mathfrak{m}}$ different subsets $\mathbf{F}(\mathfrak{n}), \mathfrak{n} \leqq \mathfrak{m}$, so the first statement of Theorem 1 is proved. The second statement follows immediately from the fact that a lower radical $\mathbf{L N}$ is hereditary if $\mathbf{N}$ is hereditary (cf. ARMendariz-LeavitT [4], Lemma 3 or Watters [9], Corollary 4).

As an immediate consequence Theorem 1 yields

L In the sense of the Zermelo-Fraenkel set theory. 
Corollary 1. If $\mathbf{P} \subseteq \mathbf{U F}$ and the subclass $\mathbf{G}$ of $\mathbf{F}$ is not a set, then for $\mathbf{Q}=\mathbf{L}(\mathbf{P} \cup \mathbf{G})$ the radicals of $[\mathbf{P}, \mathbf{Q}]$ form a class but not a set. If $\mathbf{P}$ is hereditary, then also the radicals of $[\mathbf{P}, \mathbf{Q}]$ are hereditary.

3. Here we are going to prove the existence of a unique maximal hereditary radical contained in a radical class $\mathbf{R}$ and we shall characterize it.

THEOREM 2. If $\mathbf{R}$ is an arbitrary radical class, then there exists a unique maximal hereditary radical, $\mathbf{H}_{\mathbf{R}} \subseteq \mathbf{R}$, moreover, $\mathbf{H}_{\mathbf{R}}$ is the union of all hereditary radicals $\mathbf{H} \subseteq \mathbf{R}$.

Proof. If $\mathbf{H}_{\mathbf{R}}$ is the union of all hereditary radicals contained in $\mathbf{R}$, then $\mathbf{H}_{\mathbf{R}}$ is a homomorphically closed hereditary class. Thus the lower radical $\mathbf{L H}_{\mathbf{R}}$ is hereditary and it satisfies $\mathbf{H}_{\mathbf{R}} \subseteq \mathbf{L} \mathbf{H}_{\mathbf{R}} \subseteq \mathbf{H}_{\mathbf{R}}$. Hence $\mathbf{H}_{\mathbf{R}}=\mathbf{L} \mathbf{H}_{\mathbf{R}}$ is valid.

THeOREM 3. If $\mathbf{R}$ is an arbitrary radical class, then the maximal hereditary radical $\mathbf{H}_{\mathbf{R}}$ consists of all rings each non-zero accessible subring of which has no non-zero homomorphic image in the semisimple class $\mathbf{S R}$.

Proof. As it is well-known, $\mathbf{R}$ is just the upper radical USR of the semisimple class $\mathbf{S R}$. Let $\mathbf{A}$ denote the class of all rings each non-zero accessible subring of which has no non-zero homomorphic image in the class SR. We have to show $\mathbf{A}=\mathbf{H}_{\mathbf{R}}$. The class $\mathbf{A}$ is obviously homomorphically closed and hereditary, so the lower radical $\mathbf{L A}$ is again hereditary, further $\mathbf{A} \subseteq \mathbf{L A} \subseteq$ $\subseteq \mathbf{U S R}=\mathbf{R}$ holds. Thus $\mathbf{A} \subseteq \mathbf{L A} \subseteq \mathbf{H}_{\mathbf{R}}$ follows. On the other hand, consider a ring $A \in \mathbf{H}_{\mathbf{R}}$. $A$ cannot be mapped homomorphically onto any nonzero ring of $\mathbf{S R} \subseteq \mathbf{S H}_{\mathbf{R}}$, further, since $\mathbf{H}_{\mathbf{R}}$ is hereditary, so every accessible subring of $A$ belongs to $\mathbf{H}_{\mathbf{R}}$, and it cannot be mapped homomorphically onto a non-zero ring of $\mathbf{S R}$. This means $A \in \mathbf{A}$, that is $\mathbf{H}_{\mathbf{r}} \subseteq \mathbf{A}$.

4. In Theorem 1 we have seen examples for classes $[\mathbf{P}, \mathbf{Q}]$ of radicals consisting of hereditary radicals. Generally, a class $[\mathbf{P}, \mathbf{Q}]$ of radicals may consist of non-hereditary radicals, except of $\mathbf{P}$ and $\mathbf{Q}$.

Example 1. Let $\mathbf{P}=\{0\}$ be the zero radical class, and $\mathbf{Q}$ the lower radical determined by the zero ring $Z(p)$ over a cyclic group of prime order $p$. If $\mathbf{H} \neq \mathbf{P}$ is a hereditary radical of $[\mathbf{P}, \mathbf{Q}]$, then every ring $A \in \mathbf{H}$ belongs to $\mathbf{Q}$, and so $A$ has an accessible subring isomorphic to $Z(p)$. Since $\mathbf{H}$ is hereditary, so it follows $Z(p) \subseteq \mathbf{H}$, and $\mathbf{Q}=\mathbf{L} Z(p) \subseteq \mathbf{H}$. On the other hand it is easy to see that the lower radical $Z\left(p^{\infty}\right)$ determined by the zero-ring $Z\left(p^{\infty}\right)$ over the quasicyclic group $C\left(p^{\infty}\right)$, consists exactly of rings which are (discrete) direct sums of rings isomorphic to $Z\left(p^{\infty}\right)$. Since $\mathbf{L} Z\left(p^{\infty}\right) \neq \mathbf{Q}$ so it is not hereditary. Hence $[\mathbf{P}, \mathbf{Q}]$ does not contain hereditary radicals differing from $\mathbf{P}$ and $\mathbf{Q}$, but it contains the non hereditary radical $\mathbf{L} Z\left(p^{\infty}\right)$. (Hereditary subradicals of the lower Baer-radical were treated in [3].) 
If $\mathbf{P} \subseteq \mathbf{Q}$ are arbitrary radicals, then in $[\mathbf{P}, \mathbf{Q}]$ we can define two operations. The intersection $\bigcap_{i \in I} \mathbf{R}_{i}$ of a family $\left\{\mathbf{R}_{i}\right\}_{i \in I}$ of radicals from $[\mathbf{P}, \mathbf{Q}]$ is again a radical, and it belongs to $[\mathbf{P}, \mathbf{Q}]$. If $\mathbf{R}_{1}, \mathbf{R}_{2} \in[\mathbf{P}, \mathbf{Q}]$, then the sum $\mathbf{R}_{1}+\mathbf{R}_{2}$ can be defined as the lower radical $\mathbf{L}\left(\mathbf{R}_{1} \cup \mathbf{R}_{2}\right)$. (Another representations of $\mathbf{R}_{1}+\mathbf{R}_{2}$ are given in LEAVITT [6], where it is called the join radical.) If $\mathbf{Q}$ is hereditary, then to any $\mathbf{R} \in[\mathbf{P}, \mathbf{Q}]$ the hereditary closure $\overline{\mathbf{R}}$ means the intersection of all hereditary radicals of $[\mathbf{P}, \mathbf{Q}]$. Obviously $\overline{\mathbf{R}}$ is the lower radical determined by the rings of $\mathbf{R}$ and their ideáls. It is also obvious that the hereditary closure operation in $[\mathbf{P}, \mathbf{Q}]$ is topological, in the sense that $\overline{\mathbf{R}_{1}+\mathbf{R}_{\mathbf{2}}}=$ $=\overline{\mathbf{R}}_{1}+\overline{\mathbf{R}}_{\mathbf{2}}$.

Combining these considerations with Theorem 2, it follows

COROLlaRY 2. If the radical $\mathbf{R}$ is non-hereditary, then there are hereditary radicals $\mathbf{P}$ and $\mathbf{Q}(\mathbf{P} \neq \mathbf{Q})$ such that $[\mathbf{P}, \mathbf{Q}]$ does not contain hereditary radicals except of $\mathbf{P}$ and $\mathbf{Q}$, further $\mathbf{R} \in[\mathbf{P}, \mathbf{Q}]$.

Choose, namely $\mathbf{P}=\mathbf{H}_{\mathbf{R}}$ and $\mathbf{Q}=\overline{\mathbf{R}}$.

5. Hereditary radicals appear usually by lower radical constructions. ARMendariz [2] and RJABUHIN [7] have characterized the semisimple classes having hereditary upper radicals. Here we make further investigations about the hereditariness of upper radicals.

THEOREM 4. Let $\mathbf{M}$ be a hereditary class. The upper radical $\mathbf{U M}$ is hereditary if and only if $\mathbf{U M}$ consists exactly of all rings, each non-zeroaccessible subring of which has no non-zero homomorphic image in the class $\mathbf{M}$.

Proof. By Theorem 3 the radical UM is hereditary if and only if $\mathbf{U M}$ consists of all rings each accessible subring of which has no non-zero homomorphic image in the semisimple class $\mathbf{S U M}=\overline{\mathbf{M}}$. Hence if $A \in \mathbf{U M}$, then obviously no accessible subring of $A$ can be mapped homomorphically on a ring of $\mathbf{M} \subseteq \mathbf{M}$, and if $A \notin \mathbf{U M}$, then there is an accessible subring $B$ of $A$, which has a non-zero homomorphic image $B_{1}$ in $\overline{\mathbf{M}}$, moreover, $B_{1}$ can be mapped homomorphically onto a non-zero ring of $\mathbf{M}$.

In the following two theorems we present sufficient conditions for the hereditariness of upper radical classes. In what follows, let $\mathbf{M}$ denote always a hereditary class of rings, and consider the following conditions:

(i) $\mathbf{M}$ is homomorphically closed.

(ii) If $L$ is an ideal of an ideal $I$ of a $\operatorname{ring} A$ such that $I / L \in \mathbf{M}$, then $L$ is an ideal of $A$.

(iii) If $I$ is a non-zero ideal of a $\operatorname{ring} A$ and $I \in \mathbf{M}$, then there exists a proper ideal $N$ of $A$ such that $I+N=A$.

(iv) If $I$ is an ideal of $A$ with $I \in \mathbf{M}$, and $I_{0}$ denotes the annihilator of $I$ in $A$, then $A / I_{0} \in \mathbf{M}$ follows. 
Let us recall that the (two-sided) annihilator of $I$ is defined as $I_{0}=$ $=\{x \in A \mid x I=I x=0\}$.

According to conditions (i) -(iv), observe that for instance every class of simple rings with unity element, satisfy all these conditions.

Theorem 5. If the hereditary class $\mathbf{M}$ fulfils conditions (i), (ii) and (iii), then the upper radical class $\mathbf{U M}$ is hereditary.

Proof. Suppose UM is not hereditary, and let $A \in \mathbf{U M}$ be a ring having a non-zero ideal $I \notin \mathbf{U M}$. Now $I$ can be mapped homomorphically on a non-zero ring of $\mathbf{M}$, i.e. there exists an ideal $L$ of $I$ such that $0 \neq I / L \in \mathbf{M}$. By condition (ii) $L$ is an ideal of $A$. Hence $I / L$ is an $\mathbf{M}$-ideal of $A / L$, and so by (iii) there exists an ideal $N \mid L$ of $A \mid L$ such that $A|L=I| L+N \mid L$, and $N|L \neq A| L$. This implies $I+N=A$ and $L \subseteq I, N$, so it follows

$$
0 \neq A \mid N=(I+N) / N \simeq I /(I \cap N),
$$

and $I /(I \cap N)$ is a homomorphic image of $I / L \in \mathbf{M}$. Hence by (i) we have $A \mid N \simeq I /(I \cap N) \in \mathbf{M}$, contradicting the assumption $A \in \mathbf{U M}$.

THEOREM 6. If the hereditary class $\mathbf{M}$ fulfils conditions (ii) and (iv), then the upper radical class $\mathbf{U M}$ is hereditary.

Proof. If UM is not hereditary, then there exists a ring $A \in \mathbf{U M}$ having an ideal $0 \neq I \notin \mathbf{U M}$. By Theorem 1 of ANDERson-Divinsky-Suliňski [1], the UM-radical $\mathbf{U M}(I)$ of $I$ is an ideal of $A$, and $A^{\prime}=A / \mathbf{U M}(I)$ belongs to $\mathbf{U M}$ but it has an ideal $0 \neq I^{\prime}=I / \mathbf{U M}(I)$ belonging to $\mathbf{S U M}=\mathbf{M}$. Since any nonzero ideal of $I^{\prime}$ can be mapped homomorphically onto a non-zero ring of $\mathbf{M}$, therefore there is an ideal $L^{\prime}$ of $I^{\prime}$ such that $I^{\prime} \mid L^{\prime} \in \mathbf{M}$. But $I^{\prime} \mid L^{\prime}$ is a homomorphic image of $I$, so there is an ideal $L$ of $I$ with $I\left|L \simeq I^{\prime}\right| L^{\prime} \in \mathbf{M}$. Hence condition (ii) implies that $L$ is an ideal of $A$. Consider $A^{\prime \prime}=A \mid L$ and $I^{\prime \prime}=I \mid L$. For the annihilator $I_{0}^{\prime \prime}$ of $I^{\prime \prime}$ in $A^{\prime \prime}$ conditions (iv) implies $0 \neq A|I \simeq A| L|I| L=$ $=A^{\prime \prime} \mid L^{\prime \prime} \in \mathbf{M}$, contradicting the assumption $A \in \mathbf{U M}$.

Concerning Theorem 6 we can prove that in some cases the rings of the semisimple class $\overline{\mathbf{M}}$ are subdirect sums of $\mathbf{M}$-rings. For this aim we need the concept of idealquotients. As usual, the two-sided idealquotient $L: I$ of the ideal $I \subseteq A$ is defined as the set

$$
L: I=\{x \in A \mid x I \subseteq L, I x \subseteq L\} .
$$

Obviously, if also $L$ is an ideal of $A$, then $L: I$ is an ideal of $A$. Consider the following condition

(v) If $L$ and $I$ are ideals of the ring $A$ such that $I / L \in \mathbf{M}$, then $(L: I) \cap I=L$ is valid. 
Let us remark that if $\mathbf{M}$ is a class of simple rings with unity element, then also condition ( $v$ ) is fulfilled.

THEOREM 7. If the hereditary class $\mathbf{M}$ satisfies conditions (ii), (iv) and (v) then every ring of the semisimple class $\overline{\mathbf{M}}$ is a subdirect sum of $\mathbf{M}$-rings.

Proof. Consider a ring $A \in \overline{\mathbf{M}}$. Since $A$ has a non-zero homomorphic image in $\mathbf{M}$, so there exists an ideal $M$ of. $A$ with $0 \neq A / M \in \mathbf{M}$. Let $\left\{M_{\alpha}\right\}$ be the set of all ideals of $A$ such that $A / M_{\alpha} \in \mathbf{M}$. Taking $I=\bigcap_{\alpha} M_{\alpha}$, it is sufficient to verify $I=0$. Suppose $I \neq 0$. Since $\overline{\mathbf{M}}$ is a hereditary class, so $A \in \overline{\mathbf{M}}$ implies $I \in \overline{\mathbf{M}}$. Consequently there exists an ideal $L$ of $I$ with $0 \neq I / L \in \mathbf{M}$. Hence by condition (ii) $L$ is an ideal of $A$, moreover, the annihilator of $I / L$ in $A$ is just $(L: I) / L$. Hereby condition (iv) implies

$$
A /(L: I) \cong A / L /(L: I) / L \in \mathbf{M} \text {. }
$$

Thus $(L: I) \in\left\{M_{\alpha}\right\}$ follows and so $I \subseteq(L: I)$ holds. By (v) we obtain

$$
I \subseteq(L: I) \cap \dot{I}=L \subseteq I
$$

which contradicts the choice of $L$.

Observe that condition (i) is not necessary to the hereditariness of the upper radical. Consider namely the class of all primitive rings in the sense of JACoBson. As it is well-known, this class contains primitive rings having nonzero nilpotent homomorphic images, and so this class is not homomorphically closed. On the other hand, the upper radical belonging to this class coincides with the JACOBSON-radical class which is hereditary.

Finally, in the next example we shall show that all the conditions (ii), (iii) and (iv) are not necessary for the hereditariness of an upper radical class.

ExAMPLE 2. Let $\mathbf{R}$ be the class of all rings in which the non-zero ideals are idempotent. (The VON NEULANN regular rings belong for instance to $\mathbf{R}$.) As it is well-known, $\mathbf{R}$ forms a radical class, moreover it is hereditary. The radical class $\mathbf{R}$ can be regarded as the upper radical of its semisimple class $\mathbf{S R}$. We show that none of conditions (ii), (iii) and (iv) is fulfilled by the hereditary class SR. Consider namely the algebra $A$ over the rational number field, generated by the basis elements $a_{1}$ and $a_{2}$ with the multiplication-rules $a_{1} a_{i}=$ $=a_{i} a_{1}=a_{i},(i=1,2)$, and $a_{2}^{2}=0$. Obviously the subalgebra $A_{2}=\left\{a_{2}\right\}$ is a minimal ideal of $A$, further, it is nilpotent, hence it belongs to the class SR. Condition (ii) is not satisfied, because for any proper additive subgroup $B$ of $A_{2}$ the factorring $A_{2} / B$ is again a zeroring, and so $A_{2} / B \in \mathbf{S R}$ holds, but $B$ is not an ideal of $A$, and (ii) is not fulfilled. Since $A$ has only one proper ideal, namely $A_{2}$, so there does not exist a proper ideal $N$ of $A$ such that $A_{2}+N=A$. Hence (iii) is not satisfied. At last, observe that the annihilator of $A_{2}$ is itself, but $A / A_{2}$ is isomorphic to the rational number field, and so it belongs to $\mathbf{R}$ and not to SR. Thus also condition (iv) is not valid for SR. 


\section{REFERENCES}

[1] T. Anderson, N. Divinsky and A. Sulix́ski, Hereditary radicals in associative and alternative rings, Canad. J. Math. 17 (1965), 594-603.

[2] E. P. Armendariz, Closure properties in radical theory, Pacific J. Math. 26 (1968), $1-7$.

[3] E. P. Armendariz, Hereditary subradicals of the lower Baer radical, Publ. Math. Debrecen 15 (1968), 91-93.

[4] E. P. ARmendariz and W. G. Leaviti, The hereditary property in the lower radical construction, Canad. J. Math. 20 (1968), 474-476.

[5] N. DivINsky, Rings and radicals, London, 1965.

[6] W. G. Leavite, Sets of radical classes, Publ. Math. Debrecen 14 (1967), 321-324.

[7] MI. Ju. RJaBUhIN, Radicals in categories, Mat. Issled. 2 (1967), 107 - 165 (in Russian).

[8] A. SultŃski, T. ANDERson and N. Divinsky, Lower radical properties for associative and alternative rings, J. London Math. Soc. 41 (1966), 417-424.

[9] J. F. WATtERs, Lower radicals in associative rings, Canad. J. Math. 21 (1969), $466-476$.

(Received April 25, 1970)

MTA MATEMATIKAI KUTATÓ INTÉZETE

H-1053 BUDAPEST

REÁLTANODA U. 13-15.

HUNGARY 